FORMATION Formation emploi

Revue française de sciences sociales

$110 \mid 2010$

Pêle-mêle

\title{
La construction des groupes professionnels : le cas des restaurateurs d'œuvres d'art en France et aux Etats-Unis
}

La construction des groupes professionnels: le cas des restaurateurs d'œuvres d'art en France et aux Etats-Unis

The construction of professional organizations: the case of art restorers in France and the United states

Die bildung von berufsgruppen: am beispiel von kunstrestauratoren in Frankreich und den USA

La construcción de los grupos profesionales: el caso de los restauradores de arte en Francia y en Estados Unidos

\section{Léonie Hénaut}

\section{OpenEdition}

Journals

Édition électronique

URL : http://journals.openedition.org/formationemploi/3035

DOI : 10.4000/formationemploi.3035

ISSN : 2107-0946

Éditeur

La Documentation française

Édition imprimée

Date de publication : 15 juin 2010

Pagination : 49-62

ISSN : 0759-6340

Référence électronique

Léonie Hénaut, «La construction des groupes professionnels : le cas des restaurateurs d'œuvres d'art en France et aux Etats-Unis », Formation emploi [En ligne], 110 | 2010, mis en ligne le 10 juillet 2012, consulté le 30 octobre 2020. URL : http://journals.openedition.org/formationemploi/3035 ; DOI : https://doi.org/10.4000/formationemploi.3035 


\title{
Profession
}

\section{La construction}

des groupes professionnels:

le cas des restaurateurs d'œuvres

d'art en France et aux États-Unis'

Par Léonie Hénaut ${ }^{*}$

\author{
Deux faisceaux de facteurs interviennent dans la construction \\ des dynamiques professionnelles : les institutions et les luttes professionnelles. \\ Les restaurateurs d'œuvres d'art restent indépendants en France \\ quand ils deviennent salariés aux États-Unis.
}

Le problème social auquel répond la restauration des œuvres d'art n'a pas changé depuis le XVIII ${ }^{\mathrm{e}}$ siècle : comment sauver une œuvre qui menace de disparaître, ou améliorer son état esthétique ? En revanche, le métier de restaurateur a considérablement évolué au cours $d u \mathrm{Xx}^{\mathrm{e}}$ siècle, en particulier dans les musées. Ainsi, la figure de «l'homme de métier », proche du pôle de l'artisanat avec ses savoirs empiriques transmis par apprentissage, est remplacée par celle $\mathrm{du}$ « professionnel », au sens anglo-saxon du praticien qui mobilise des savoirs scientifiques après avoir suivi une formation spécialisée. Là où n'existaient que des formations par apprentissage en atelier, on assiste en effet à l'apparition de formations au sein

1 Cet article est issu d'une recherche doctorale et postdoctorale sur la restauration des œuvres d'art. Les données portant sur les États-Unis ont été recueillies à l'occasion d'un séjour postdoctoral à New York en tant que visiting scholar à la New School for Social Research.
* Léonie Hénaut est docteure en sociologie, postdoctorante au Centre de sociologie de l'innovation (Mines-ParisTech/CNRS). Ses travaux portent sur l'interdépendance des groupes professionnels impliqués dans un même champ d'activité, en l'occurrence la conservation des œuvres de musées et, plus récemment, les soins et services au domicile des personnes âgées et handicapées. Elle a publié récemment : "L'interprétation comme activité : matérialité et signification d'une œuvre ", Sociologie de l'art, n 14, 2009 ; "Montée en qualification et perte de contrôle. Les restaurateurs de tableaux et leurs documents de travail ", in Observer le travail. Histoire, ethnographie, approches combinées, Arborio A.-M., Cohen Y., Fournier P., Hatzfeld N., Lomba C. et Muller S. (Dir.), Paris, La Découverte, Collection "Recherches », 2008 ; "Polymères et vieilles dentelles. La restauration et la conservation dans un musée de costumes ", Sociétés contemporaines, nº 66, 2007. 
d'établissements de l'enseignement supérieur, délivrant des cours théoriques en histoire de l'art, chimie et biologie, et promouvant de nouveaux standards de restauration et de conservation. Il s'agit désormais, par exemple, de faire une analyse préalable de l'œuvre, de documenter les interventions réalisées par des photographies et des comptes-rendus, de choisir dans la mesure du possible un traitement réversible, ou encore de se soucier des conditions hygrométriques d'exposition des œuvres.

Les diplômés de ces formations entament partout un processus de professionnalisation, au sens où ils aspirent à voir leur expertise davantage reconnue et tendent à s'organiser sur le modèle des professions établies. Cependant, ce processus n'emprunte pas le même chemin dans tous les contextes nationaux. Nous avons choisi de comparer les cas des restaurateurs d'œuvres d'art français et américains car ils incarnent deux modèles de développement professionnel particulièrement contrastés, voire inversés.

En France, les diplômés issus des quatre formations spécialisées, créées à partir des années $70^{2}$, sont environ 1000 à être en activité aujourd'hui, la plupart exerçant sous le statut de profession libérale ${ }^{3}$. Ils ont obtenu la fermeture du marché des musées en leur faveur au terme d'une lutte avec les restaurateurs de formation traditionnelle ${ }^{4}$, mais souhaiteraient que des

\footnotetext{
${ }^{2}$ La maitrise de sciences et techniques en conservation-restauration des biens culturels (MST, aujourd'hui Master CRBC), créée à l'université Paris 1 en 1973 ; l'Institut de formation des restaurateurs d'œuvres d'art (IFROA, aujourd'hui intégré à l'Institut national du patrimoine qui forme également les conservateurs), créé par le ministère de la Culture en 1978 ; la section restauration d'œuvres sculptées de l'École régionale des beaux-arts de Tours, créée en 1982 ; la section conservation-restauration des œuvres peintes de l'École municipale des beaux-arts d'Avignon pour la peinture, créée en 1983.

${ }^{3}$ Une enquête par questionnaires, réalisée en 1993 par le département des Études et de la Prospective du ministère de la Culture, révèle que la population des restaurateurs d'œuvres d'art, toutes formations confondues, compte, en France, plus de 4500 praticiens, dont $61 \%$ sont artisans. Voir notamment : « La profession de restaurateur d'œuvres d'art », Développement culturel, Bulletin du département des Études et de la Prospective, ministère de la Culture et de la Francophonie, n ${ }^{\circ}$ 104, juin 1994, pp. 1-6.

${ }^{4}$ Depuis la «Loi Musées », votée en 2002, seuls les restaurateurs ayant l'un des quatre diplômes précédemment cités sont autorisés à intervenir sur les œuvres des musées de France, et donc à répondre aux appels d'offres formulés par les musées. Les non-diplômés qui travaillaient pour les musées ont néanmoins pu obtenir une habilitation. $C f$. « Décrets du 25 avril 2002 pris pour l'application de la loi $n^{\circ} 2002-5$ du 4 janvier 2002 relative aux musées de France », Titre III, Chapitre 2, Article 13.
}

postes spécifiques soient créés au sein des musées et que leur expertise en situation de travail soit davantage reconnue, par les conservateurs de musées notamment. Aux États-Unis, au contraire, l'activité n'est toujours pas réglementée alors que les premières formations apparaissent dès les années 60 ; cependant, les restaurateurs diplômés - ils sont aussi un millier environ - sont généralement salariés des musées et jouissent d'un niveau d'autorité et de rémunération comparable à celui des conservateurs 5 .

Pour expliquer cet écart, il est tentant de retenir une explication de type culturaliste, ou un facteur explicatif unique comme le degré d'interventionnisme de l'État. Nous considérons que la construction des groupes professionnels, et partant la différenciation des chemins de professionnalisation qu'ils empruntent selon les pays, ne se joue pas seulement au niveau de l'État et des associations professionnelles, mais aussi dans les interactions et les luttes entre les différentes catégories de professionnels impliquées dans le champ d'activité. Ce faisant, notre approche s'inspire tout autant des travaux d'Elliot Freidson (2001), qui souligne le rôle des institutions, que du cadre théorique proposé par Andrew Abbott (1988), pour qui la dynamique des professions tient essentiellement aux luttes professionnelles. En l'occurrence, il s'agit ici des luttes qui s'exercent entre des restaurateurs d'œuvres d'art de formation et de génération différentes, mais aussi entre des restaurateurs et d'autres corps de métier avec lesquels ils sont amenés à coopérer : les conservateurs, mais aussi les régisseurs s'occupant du transport des œuvres, ou encore les scientifiques de laboratoire chargés de les analyser.

En partant du cas des restaurateurs et en utilisant les premiers résultats d'une comparaison internationale, l'objectif de cet article est moins d'expliquer les différences observées terme à terme entre les deux dynamiques professionnelles que de montrer le caractère sociétal de la construction des professions, en soulignant la diversité des facteurs et des niveaux de réalité

\footnotetext{
${ }^{5}$ Les résultats des nombreuses enquêtes réalisées chaque année par différentes associations convergent tous en ce sens. L'enquête de l'association américaine des directeurs de musées indique ainsi un salaire médian annuel de 52224 à 87125 dollars (soit de 39575 à 66000 euros) pour un conservateur (curator), selon sa position hiérarchique, et de 51480 à 88300 dollars (39 000 à 67000 euros) pour un restaurateur (conservator). Voir: Association of Art Museum Directors, Salary survey, New York, 2008.
} 
impliqués - les institutions, les luttes interprofessionnelles et les interactions sur le lieu de travail. Nous mettrons en évidence le rôle de ces facteurs après avoir présenté plus en détail les processus qui caractérisent les développements professionnels des restaurateurs français et américains.

\section{Encadré 1}

\section{Méthodologie}

Pour la France, une quarantaine d'entretiens a été réalisée avec des restaurateurs d'œuvres d'art, des étudiants en restauration, des conservateurs de musées et des représentants des groupements professionnels du domaine. Les bulletins d'associations et les revues spécialisées publiés depuis les années 50 ont également été dépouillés de façon exhaustive. Dans cet article, nos analyses s'appuient aussi sur des données chiffrées recueillies auprès de la direction des Musées de France, ou bien présentes dans des rapports d'enquête réalisées par les ministères de la Culture et du Commerce. On se réfère notamment à l'enquête par questionnaires réalisée par le département des Études et de la Prospective du ministère de la Culture sur la population des restaurateurs en 1993.

Aux États-Unis, une dizaine de restaurateurs en exercice ont été interviewés et la documentation professionnelle a été consultée, de même que les documents produits par les formations spécialisées. Nous avons notamment utilisé les listes des diplômés qui en sont issus pour décrire statistiquement la population en termes de flux, de sexe, de lieu d'activité et de statut d'exercice professionnel. Les transformations touchant à l'activité et au personnel d'une dizaine de musées ont pu être appréhendées grâce au dépouillement des rapports annuels d'activité rédigés depuis les années 40. En particulier, nous avons étudié le cas du Metropolitan Museum of Art à New York, qui est celui qui offre le plus de points communs avec le musée du Louvre étudié pendant la préparation de notre thèse, en particulier quant au rôle moteur joué dans la régulation de l'activité de restauration des œuvres d'art.

\section{FRANCE/ÉTATS-UNIS : DES CHEMINS DE PROFESSIONNALISATION INVERSÉS}

Aujourd'hui, les restaurateurs d'œuvres d'art français exerçant pour les musées évoquent leurs homologues américains comme un modèle, enviant tantôt leur statut de salariés des musées, tantôt leur capacité à produire des connaissances scientifiques et à tester de nouveaux procédés. De leur côté, les restaurateurs américains interviewés s'étonnent de la quasi-absence des restaurateurs français dans les conférences internationales ou de leur résistance à adopter certaines pratiques de conservation dites non intrusives, par exemple dans le domaine de la restauration du mobilier. Ces dernières remarques véhiculent à tort l'idée d'un retard des restaurateurs français dans leur développement professionnel. Les praticiens américains des années 50 ne sont pas l'équivalent des restaurateurs français des années 80 , et il n'y aucune nécessité à ce que les restaurateurs français aient un jour les mêmes conditions d'exercice que les restaurateurs américains d'aujourd'hui. Les similitudes et les écarts entre les histoires propres aux restaurateurs français et américains font l'objet de notre étude.

\section{Des éléments de professionnalisation communs}

Les deux groupes partagent certains processus caractérisant leur dynamique professionnelle : l'organisation en associations, la spécialisation et la féminisation. La première association qui rassemble des restaurateurs américains est fondée en 1950, à l'initiative de ceux qui sont considérés aujourd'hui comme les fondateurs de la conservation moderne : un restaurateur et un chimiste exerçant depuis les années 30 au sein du laboratoire de conservation du Fogg Art Museum, associé à l'Université d'Harvard. Cette association, appelée International Institute of Conservation (IIC), a une vocation internationale et est ouverte aux conservateurs de musées. Mais un petit groupe de restaurateurs qui se sont formés - le plus souvent sur le tas - à l'usage des instruments scientifiques d'analyse des œuvres et qui utilisent des savoirs théoriques se servent de l'IIC comme d'une 
tribune, dès les années 50 . Ils se nomment conservators pour se distinguer des restorers ne jurant que par les méthodes artisanales, et revendiquent la dimension intellectuelle et la spécificité de leur expertise. Après avoir longtemps fonctionné comme un groupe de l'IIC, l'American Institute of Conservation (AIC) s'autonomise en 1972. C'est essentiellement dans ce cadre que les restaurateurs américains s'organisent par la suite, avec la rédaction d'un code d'éthique et l'organisation de manifestations scientifiques.

En France, les restaurateurs ne commencent à se mobiliser qu'à partir du début des années 80 , à travers plusieurs associations qui se rassemblent en 1990 en une coordination d'associations, puis en une fédération de membres individuels, aujourd'hui Fédération française des professionnels de la conservationrestauration (FFCR). Avant cela, quelques restaurateurs français adhéraient à des organisations syndicales artisanales regroupant d'autres corps de métiers comme les tapissiers ou les encadreurs mais ils étaient très minoritaires. L'organisation des praticiens en association marque donc la fin d'une longue période d'isolement ainsi que l'avènement d'un nouveau type d'aspirations que l'on pourrait qualifier de professionnelles dans la mesure où elles sont tournées vers la reconnaissance de la dimension intellectuelle et spécialisée de l'activité exercée et non plus vers la défense des intérêts « économiques » et « directement matériels » du corps de métier, qui caractérise généralement les organisations professionnelles artisanales (Zarca, 1988, p. 248) .

L'apparition de formations spécialisées en restauration, de type universitaire, là où n'existaient que des formations de type artistique ou artisanal, transforme fondamentalement le champ d'activité, et cela dans les deux pays. Aux États-Unis, les premières formations spécialisées sont créées une décennie plus tôt qu'en France : la première ouvre en 1960 au sein de l'Institut des beaux-arts de l'université de New York, puis d'autres dans les années 70 , également au sein d'universités, alimentant la population des diplômés à raison de 25 personnes environ par an. Les deux

\footnotetext{
${ }^{6}$ Jusqu'au début des années 80 , les discussions qui ont lieu au sein des associations et des syndicats existants touchent par exemple aux tarifs, aux conditions pour assurer la formation des apprentis en atelier, ou encore à la nécessité de contrôler les effectifs de praticiens en exercice.
}

principales formations françaises apparaissent dans les années 70 : la première avec la création, en 1973, d'une maîtrise de sciences et techniques en conservation-restauration des biens culturels à l'université Paris 1, et la seconde, en 1978, avec la création d'un Institut de formation des restaurateurs d'œuvres d'art relevant du ministère de la Culture. Toutes formations confondues, le flux de nouveaux diplômés français varie entre 40 et 50 personnes environ chaque année.

Ce processus de spécialisation s'accompagne, enfin, de la féminisation du groupe dans les deux cas. En France, dans les années 90, les praticiens diplômés des nouvelles formations sont des femmes pour les trois-quarts, quand la population totale des restaurateurs en exercice compte seulement un tiers de femmes. Aux États-Unis, on observe, en quarante ans, une féminisation de la population des diplômés : les premières promotions des années 60 sont féminines à $40 \%$ alors que celles d'aujourd'hui le sont à $90 \%$. Une telle féminisation doit autant à la féminisation qui touche les métiers du secteur culturel qu'à celle qui s'observe, plus discrètement, dans les activités scientifiques et techniques (Marry, 2004). Ainsi, la morphologie et l'organisation du groupe des restaurateurs ont bien changé depuis les années 60 , et cela dans les deux pays.

\section{Des dynamiques professionnelles inversées}

Au-delà des similitudes évoquées, d'autres processus caractérisant la dynamique du groupe des restaurateurs américains ne touchent pas le groupe des restaurateurs français, et inversement. Les trois processus pour lesquels le contraste est le plus frappant sont la réglementation de l'activité, l'évolution du statut professionnel (indépendant ou salarié), et l'autonomie effective dont jouissent les restaurateurs dans leur travail. Comme nous l'avons résumé dans le tableau suivant, nous avons affaire à deux chemins de professionnalisation inversés.

La première différence majeure entre les deux pays touche à la réglementation de l'exercice de l'activité. $\mathrm{Si}$, en France, le marché de la restauration des œuvres de musées est légalement fermé aux non-diplômés des nouvelles formations depuis la «Loi Musées » de 2002, il n'existe aucune réglementation aux 
Tableau 1

Les restaurateurs diplômés des formations spécialisées en France et aux États-Unis

\begin{tabular}{|c|c|}
\hline France & États-Unis \\
\hline $\begin{array}{c}\text { Monopole sur le marché des musées } \\
\text { (Loi Musées de 2002) }\end{array}$ & Pas de réglementation \\
\hline $85 \%$ d'indépendants & $65 \%$ de salariés des musées ou bibliothèques \\
\hline $\begin{array}{c}\text { Faible présence dans les congrès } \\
\text { et les revues internationales }\end{array}$ & $\begin{array}{c}\text { Production scientifique, présence forte dans les congrès } \\
\text { et les revues internationales }\end{array}$ \\
\hline $\begin{array}{c}\text { Lutte pour le contrôle de l'intervention } \\
\text { Pas d'autonomie dans l'organisation du dravail l'intervention }\end{array}$ \\
\hline
\end{tabular}

États-Unis. L'instauration d'une procédure de certification des praticiens par l'AIC fait pourtant débat au sein de l'association elle-même, depuis les années 80 , et vient de connaître un regain d'actualité. Il est question d'instaurer plutôt un système d'accréditation des formations. Mais dans les deux cas, le groupe connaîtrait une réglementation très différente de celle en vigueur en France : la certification serait maîtrisée par l'association professionnelle et ne pourrait agir que comme un signal à l'endroit des clients ou des musées lors des recrutements alors que la "Loi Musées» empêche rigoureusement les praticiens non habilités d'intervenir sur les collections des Musées de France.

En second lieu, la montée en qualification des restaurateurs s'accompagne du développement massif $\mathrm{du}$ salariat au sein des organisations culturelles aux États-Unis, quand les musées français continuent de faire appel à des prestataires indépendants par le biais d'appels d'offres. À partir des années 60, en effet, les musées américains commencent à recruter du personnel sur des postes de restaurateurs et non plus sur des postes indifférenciés d'assistants ou de techniciens. Les diplômés des formations spécialisées, notamment, sont nombreux à travailler au sein d'une institution plutôt qu'à leur compte : aujourd'hui, environ $35 \%$ des diplômés exercent en pratique privée (patron, associé ou salarié d'une entreprise individuelle), $5 \%$ sont salariés d'une université, $55 \%$ d'une organisation culturelle conservant des collections (musée, bibliothèque, maison historique, centre d'archives), et $5 \%$ d'un centre régional de conservation à but non lucratif. Notons que la plupart des musées américains possèdent leurs propres ateliers de restauration ou s'adressent aux centres régionaux, à l'inverse de la situation française où la plupart des musées n'ont pas d'atelier de restauration intégré et où les travaux de restauration sont réalisés par des restaurateurs indépendants dans des services nationaux ou interrégionaux offrant leurs services à plusieurs musées. Ainsi, en France, $5 \%$ des restaurateurs sont certes salariés d'une organisation publique (musées ou bibliothèques) mais la plupart des travaux de restauration opérés sur les œuvres de musées sont réalisés par des restaurateurs indépendants, exerçant sous le statut d'artisan ou de profession libérale.

L'une des conséquences directes de cette différence en termes de statut s'observe dans le fait qu'aux ÉtatsUnis, on assiste à l'apparition d'un segment que l'on peut appeler « savant» ou « académique » (Freidson, 1986), dans la mesure où il est constitué de restaurateurs spécialisés ne pratiquant plus ou très peu la restauration et dont l'activité est pleinement vouée à la recherche scientifique ou à l'enseignement au sein d'universités ou d'institutions culturelles. Cette segmentation du groupe n'a pas lieu en France, où tous les restaurateurs se définissent par leur qualité de praticien, même lorsqu'ils enseignent au sein des formations spécialisées. La faible présence des restaurateurs français dans les congrès internationaux et les revues scientifiques peut être mise sur le compte de leur statut d'indépendants, qui ne leur permet pas de réaliser beaucoup de recherches. Mais au-delà, elle est aussi liée au fait que, lorsque les travaux de restauration donnent lieu à des conférences ou des publications, ce sont souvent les conservateurs de musées qui les présentent. Plusieurs restaurateurs ont ainsi mentionné avoir été " pillés » par des conservateurs qui ont publié des articles sur les travaux de restauration sans citer le praticien qui avait réalisé le travail et rédigé le rapport de restauration. 
La dernière différence entre les restaurateurs français et américains a trait à leur autonomie professionnelle, entendue ici comme la maîtrise concrète de l'organisation de leur activité et du déroulement des interventions de restauration. En France, les services de restauration des musées sont administrés par des conservateurs fonctionnaires, historiens d'art de formation. Ce sont eux qui organisent le travail, au sens où ils collectent les demandes des musées, font établir des devis, planifient et suivent le travail. Les conservateurs interviennent aussi dans les choix de restauration et peuvent même être amenés à refuser la solution proposée par le restaurateur et confier le travail à un autre, comme s'ils étaient substituables. Tout cela paraît impensable outre-Atlantique, où les restaurateurs dirigent les services de restauration, et où les conservateurs n'ont qu'un rôle consultatif dans les décisions d'intervention. Le développement du salariat des restaurateurs s'est donc opéré dans le sens d'une division du travail plutôt horizontale avec les conservateurs.

L'écart entre les deux dynamiques ne tient pas au seul statut professionnel - indépendant ou salarié. Ce n'est pas parce que les restaurateurs américains sont pleinement insérés dans une division du travail qu'ils contrôlent davantage leur activité. En effet, jusque dans les années 70 , les restaurateurs français qui intervenaient en tant que prestataires indépendants sur les peintures des musées organisaient eux-mêmes le travail réalisé au sein des ateliers centraux (Hénaut, 2008). Et aujourd'hui, les rares restaurateurs diplômés salariés exercent au contraire très souvent sous la direction d'un conservateur.

La comparaison invite donc à questionner le lien entre les conditions d'exercice d'une activité et l'autonomie professionnelle des praticiens. Terence Halliday (1987) montre ainsi que les architectes exerçant en tant qu'indépendants maîtrisent davantage leur activité que les ingénieurs salariés des grandes firmes, là où nos recherches invitent à penser que les restaurateurs américains salariés des musées sont plus autonomes que leurs homologues français indépendants. Le cas français révèle aussi que l'obtention d'un monopole par un groupe professionnel ne s'accompagne pas nécessairement d'un accroissement de l'autonomie de ses membres en situation de travail. L'évolution du statut professionnel et l'obtention d'un monopole ne sont pas nécessairement concomitantes d'un accroissement de l'autonomie effective dont disposent les praticiens dans l'exercice concret de leur activité.

Bien qu'ayant des trajectoires différentes, les deux groupes étudiés détiennent donc incontestablement des éléments de professionnalisation. Cependant, si on se réfère au modèle professionnel tel qu'il est classiquement décrit en sociologie des professions (Dubar et Tripier, 1998), on peut s'étonner de la distribution de ces éléments : monopole sur le marché des musées, indépendance mais faible contrôle sur l'intervention côté français ; non-réglementation de la profession, salariat mais autonomie dans l'exercice de l'activité et contrôle de l'organisation du travail côté américain. Comment expliquer une telle diversité des voies de professionnalisation ? La comparaison internationale permet de mettre en évidence les processus qui alimentent la construction des groupes professionnels.

Dans les deux sections qui suivent, il ne s'agit pas d'analyser, de façon exhaustive, tous les facteurs intervenant dans cette construction mais de les identifier, en soulignant leur rôle respectif. L'idée défendue ici est qu'il y a deux faisceaux de facteurs intervenant différemment : d'une part, les institutions, comme l'État et les associations professionnelles, qui contribuent à déterminer les contours possibles de la régulation des professions, c'est-à-dire la forme que prend leur dynamique ; d'autre part, les luttes interprofessionnelles et les interactions sur le lieu de travail, qui alimentent concrètement cette régulation, c'est-à-dire qui sont à l'origine de la dynamique effective du groupe professionnel.

\section{COMMENT LES INSTITUTIONS DÉTERMINENT LES PROCESSUS DE PROFESSIONNALISATION}

\section{L'État et ses figures de substitution}

Pour la plupart des sociologues des professions, le déterminant essentiel des processus de professionnalisation est l'État (Le Bianic, 2008). Arnold Heidenheimer (1989) a ainsi proposé de distinguer les pays selon l'importance du développement de 
leur État (« stateness »), c'est-à-dire le degré auquel les institutions étatiques sont centralisées, coordonnées hiérarchiquement, distinctes des institutions de la société civile, et interviennent dans les affaires politiques, économiques et sociales. Il est par exemple communément admis que la France a un degré de développement étatique fort, là où celui des États-Unis est faible. Mais cette opposition diamétrale peut masquer bien des nuances. Un pays peut par exemple avoir un État fortement centralisé ou interventionniste dans un domaine comme la santé ou l'éducation, et peu interventionniste dans un autre comme la culture. Surtout, rien ne permet de conclure a priori à l'impact du degré de centralisation d'un État sur la forme d'une dynamique professionnelle. Ce qu'il faut retenir de l'approche de Heidenheimer, comme le rappelle Freidson (2001, p. 135), c'est l'intérêt d'analyser les variations dans l'organisation des États ou dans le type de politiques mises en œuvre, et d'étudier dans quelle mesure ces différences pèsent sur les groupes professionnels.

L'intervention de l'État dans le développement professionnel des restaurateurs, en France, ne se limite pas à la création des formations spécialisées dans les années 70 et à la réglementation du marché des musées en 2002, soit à ce qu'on peut considérer comme les deux principaux jalons du processus de professionnalisation. Elle se manifeste pendant tout le processus, à travers la multitude de figures qui incarnent les pouvoirs publics, à commencer par les musées. La politique de rénovation et de développement dont ils font l'objet, dès la fin des années 70, a sans doute été déterminante, avec la «Loi programme sur les musées », et, surtout, dans les années 80 , l'essor du budget du ministère de la Culture ; cette politique s'est alors accompagnée d'une forte augmentation des crédits de restauration et donc de la demande de maind'œuvre qualifiée pour effectuer les travaux sur les collections publiques. En première approche, le cas des restaurateurs d'œuvres d'art s'inscrit pleinement dans le schéma français classique de professionnalisation dans lequel «l'État est [...] un acteur puissant et actif dans le lancement d'expériences éducatives destinées à former des spécialistes, notamment ceux dont il a besoin pour son importante fonction publique et pour les services publics dont il se porte garant. " (Freidson, 1994, pp. 120-121) Mais le cas étudié révèle surtout que le rôle de l'État est loin d'être unidirectionnel. Ainsi, au moment de la préparation de la
«Loi Musées », les instances ministérielles représentatives des Métiers d' $\operatorname{art}^{7}$ et de l'Artisanat soutiennent les restaurateurs de formation traditionnelle quand la direction des Musées de France travaille à ce que l'activité soit partout réservée aux seuls diplômés. La figure de l'État peut donc être déconstruite en plusieurs figures dont les rôles sont parfois contradictoires, mais qui toutes infléchissent le développement professionnel des restaurateurs.

Si l'État américain est parfois venu en aide aux musées, comme dans les années 70 , ces derniers bénéficient généralement de très peu de financements publics et ne font pas l'objet de politiques spécifiques (Martel, 2006). La perspective comparative permet néanmoins de questionner ce qui, en l'absence d'institutions publiques, a pu jouer un rôle analogue dans le développement des restaurateurs d'œuvres d'art. Prenons par exemple la création des formations spécialisées. En France, les deux plus importantes formations peuvent être vues comme le seul fruit de l'application de politiques : la création de la MST s'inscrit ainsi dans l'application des directives du «VIe Plan de développement économique et social » encourageant les universités à développer des filières professionnelles, et celle de l'IFROA (Institut français de restauration des œuvres d'art) est l'opération-phare du programme développé par Valéry Giscard-d'Estaing pour revaloriser les « métiers d'art ». Aux États-Unis, il en va complètement différemment : créée à l'initiative de restaurateurs formés dans le laboratoire pionnier du Fogg Art Museum, la première formation est entièrement financée par la fondation Rockefeller. Dès le départ, les restaurateurs américains n'ont donc aucun lien avec les pouvoirs publics, ce qui n'a sans doute pas favorisé la règlementation du groupe ensuite.

\section{L'impact de la composition des organisations professionnelles}

Si l'organisation et le degré d'interventionnisme de l'État et des multiples figures qui mettent en œuvre ses politiques représentent des éléments de contingence

\footnotetext{
${ }^{7}$ Le terme désigne une branche professionnelle qui apparaît à la fin $\mathrm{du} \mathrm{XIX}^{\mathrm{e}}$ siècle et bénéficie depuis d'une attention particulière, quoique irrégulière, de la part de l'État (Rebérioux, 1990). Depuis le milieu des années 70, les métiers d'art font l'objet d'une politique de soutien de la part de la direction de l'Artisanat, et plus irrégulièrement de la part du ministère de la Culture.
} 
particulièrement pesants pour la professionnalisation, il en va de même de l'organisation et de la représentation des groupes professionnels. Là où la plupart des auteurs étudient les actions menées par les associations professionnelles auprès des pouvoirs publics pour accroître leur contrôle sur l'activité, Freidson (2001, p. 142) propose de s'intéresser également aux groupes, non systématiquement organisés en associations, constitués par les praticiens ayant suivi une même formation. Ces derniers peuvent en effet nourrir des aspirations qui ne recouvrent pas celles portées par les associations représentant officiellement la profession. Ils peuvent donc être considérés comme des acteurs à part entière du développement du groupe professionnel. La comparaison des cas américain et français permet d'étudier différentes relations entre les associations professionnelles et les autres communautés d'intérêt et de mettre en évidence l'impact de ces relations sur la dynamique professionnelle du groupe considéré.

Les premières associations sont, dans les deux cas, créées à l'initiative d'une petite élite de restaurateurs d'œuvres d'art, plus qualifiés que la moyenne des praticiens de leur époque, qui cherchent à revaloriser leur activité. En revanche, rapidement, l'association professionnelle représentant les restaurateurs français se limite quasiment aux seuls diplômés des nouvelles formations françaises et étrangères - la FFCR (Fédération française des conservateursrestaurateurs) en rassemble plus de 300 sur les 1000 diplômés en activité. Au contraire, celle qui représente les restaurateurs américains reste ouverte à tous les praticiens souscrivant au code de déontologie élaboré par l'association, si bien que sur les 3300 membres de l'AIC (American Institute of Conservation), moins de 1000 sont diplômés. Dans quelle mesure l'ouverture des associations professionnelles aux non-diplômés favorise-t-elle la professionnalisation d'un groupe professionnel?

Dans une première phase, l'ouverture de l'association américaine à tous les restaurateurs a pu donner au groupe un plus grand poids dans les négociations visant à faire reconnaître la spécificité de l'activité et l'expertise des praticiens, notamment auprès des directeurs de musées. En revanche, par la suite, cette même ouverture a freiné le processus de certification et, plus généralement, la conquête de la protection du marché par les diplômés. En effet, les débats qui ont lieu au sein de la profession, depuis les années 80 , montrent qu'à chaque fois qu'est proposée la mise en place d'un processus de certification des restaurateurs, ou d'accréditation des programmes de formation, les non-diplômés, qui ont pourtant accédé à d'importantes positions et sont reconnus par leurs collègues, s'y opposent en soulignant les dangers de l'exclusion et de la standardisation des formations. Dans le cas français, il est certain que la fermeture de la FFCR aux non-diplômés a favorisé l'obtention d'une réglementation du marché des musées. En revanche, elle rend aujourd'hui très difficile la duplication de la réglementation aux autres marchés - les antiquaires et les particuliers, mais aussi les Monuments historiques - sur lesquels les restaurateurs de formation traditionnelle sont les plus nombreux. La composition des associations professionnelles a donc un impact sur la trajectoire des groupes professionnels.

Pour conclure cette partie, nous ne considérons pas que les institutions déterminent directement le développement professionnel des restaurateurs d'œuvres d'art, au sens où elles seraient à l'origine de tel ou tel développement, mais plutôt qu'elles définissent les conditions dans lesquelles la dynamique de professionnalisation peut avoir lieu. Ainsi, l'interventionnisme de l'État français en matière de restauration des collections publiques crée des conditions favorables à la réglementation du marché des musées. De même, l'ouverture de l'association des restaurateurs américains aux non-diplômés rend peu probable l'obtention d'un monopole par les seuls diplômés quand sa fermeture la rend au contraire possible dans le cas français. Pour compléter cet examen des institutions qui rendent ou non possibles tel ou tel chemin de professionnalisations, telle ou telle autre forme de dynamique professionnelle, il convient donc d'étudier maintenant les mécanismes qui produisent effectivement les chemins observés. 


\section{COMMENT LES LUTTES PROFESSIONNELLES ALIMENTENT LA CONSTRUCTION DES PROFESSIONS}

Empruntant à Andrew Abbott (1988) son cadre d'analyse des professions, nous n'appréhendons pas la dynamique professionnelle des restaurateurs d'œuvres d'art de façon monographique, mais en faisant l'hypothèse qu'elle est dépendante des dynamiques suivies par les autres groupes impliqués dans le même « champ d'activité »-ici la conservation des œuvres de musées. Ces autres groupes sont les conservateurs (curators), que nous avons déjà évoqués, historiens d'art de formation et principalement chargés de l'organisation des expositions ; les régisseurs qui s'occupent principalement du transport des œuvres ; ou les scientifiques de laboratoire, qui les analysent. Ces groupes professionnels ne font pas seulement système parce qu'ils coexistent et peuvent s'affronter lorsque les frontières entre leurs activités sont floues, mais aussi parce que leurs développements respectifs sont liés quand un groupe professionnel gagne en prestige ou en autorité, un autre en perd. Dans quelles conditions, et par quels mécanismes, les luttes professionnelles alimentent-elles la construction des professions? Nous allons voir, d'abord, qu'elles transforment le contenu de l'activité vers plus d'abstraction; ensuite, qu'elles alimentent ou non le développement du salariat des restaurateurs ; enfin, que les négociations qui ont lieu au sein des organisations - ici les musées - peuvent être à l'origine de la dynamique d'une profession tout entière.

\section{Des luttes professionnelles qui transforment le contenu de l'activité...}

Le premier type de luttes professionnelles, entre restaurateurs d'œuvres d'art de formation et de génération différentes, se solde par l'exclusion des restaurateurs de formation traditionnelle du marché des musées et par la redéfinition de l'activité de restauration comme « scientifique » et non plus « artisanale». Dans tous les domaines, et d'abord en peinture, on assiste au même processus : un petit groupe de praticiens dotés de connaissances scientifiques revendique de nouvelles méthodes, fondées sur des sciences exactes, et condamne les savoirs informels et les procédés traditionnels qui jusque-là faisaient l'unanimité. Ensuite, des affrontements ont lieu par articles ou ouvrages interposés, ou bien lors de conférences internationales. En matière de restauration du support des peintures sur toile, par exemple, les premières tensions s'observent dans les années 60-70, en Angleterre, entre les partisans des analyses chimiques et de la table chauffante sous vide d'air (appelés alors « les Mods ») et ceux qui défendent le rentoilage traditionnel à la colle (« les Trads») (Percival-Prescott, 2003). Les débats techniques masquent l'enjeu purement professionnel qui motive les participants : le gain ou le maintien sur le marché et au sein des organisations. Dans tous les domaines, ce type d'affrontements Trads/Mods contribue à la transformation du contenu de l'activité vers davantage d'abstraction.

La comparaison franco-américaine révèle le plus souvent un décalage temporel entre les deux pays. Le domaine de la restauration des sièges, par exemple, était, jusqu'au début des années 2000, complètement dominé par des tapissiers qui dégarnissent généralement les sièges pour les doter d'une garniture et d'étoffes neuves. La poignée de restaurateurs français sensibilisés aux nouvelles pratiques, moins intrusives, lors de stages ou de colloques, commencent à peine à imposer leur approche alors que leurs homologues américains l'ont fait il y a vingt ans sans rencontrer une grande résistance. Les Français ont d'autant plus de difficultés que les tapissiers sont bien plus nombreux, très organisés professionnellement, et soutenus par les pouvoirs publics.

\section{... et qui alimentent les dynamiques professionnelles}

Si on insiste souvent sur les luttes qui ont lieu entre des groupes professionnels ou des sous-groupes concurrents sur un marché du travail, comme on vient de le faire, notre étude montre aussi que les luttes professionnelles sont particulièrement durables et virulentes lorsqu'elles touchent des groupes qui sont amenés à coopérer. Aux États-Unis comme en France, les premières revendications des restaurateurs s'exercent en effet contre les conservateurs de musées qui cherchent à réduire leur rôle à celui de simples 
exécutants. Dans un article adressé en 1950 aux conservateurs de musées américains, l'un des fondateurs de l'IIC et principal acteur du mouvement écrit ainsi : "L'homme qui fait le travail est celui qui doit savoir ce qu'il fait. [...] Quand vous n'en faites, le plus souvent, qu'un bouf qui doit être conduit, vous retirez du schéma de l'opération tout espoir de contrôle responsable et sensible. Si l'érudit, le surveillant qui se tient à côté de l'ouvrier sait parfaitement ce qui devrait être fait, alors selon toutes méthodes de raisonnement, c'est le surveillant lui-même qui devrait faire le travail. » (Stout, 1950) Bien plus tard, la question de l'autonomie professionnelle est aussi au cœur des premières revendications des restaurateurs français. C'est même la seule à être commune à tous les praticiens, quels que soient leur statut juridique, leur âge et leur formation initiale. Lors d'une réunion de leur association en 1987, les diplômés de l'IFROA réagissent ainsi aux propos tenus par un conservateur lors d'un forum en soulignant: «Un bon restaurateur [...] n'est pas seulement "l'exécutant" mais aussi le "conseilleur" et le "spécialiste" dont le conservateur et le scientifique doivent écouter les avis. ${ }^{8}$

On observe d'ailleurs un « processus mimétique » (DiMaggio et Powell, 1983) chez les restaurateurs français qui prennent les revendications et les écrits des premiers conservators américains comme modèles. Lorsqu'ils élaborent une première charte, au début des années 80 , ils s'appuient également sur le code canadien alors en cours d'élaboration, notamment parce que l'un des étudiants de la MST présent lors des réunions inter-associations venait de suivre un stage dans un institut de conservation canadien renommé. Ainsi, la fréquentation d'organisations où les restaurateurs ont une plus grande autonomie de même que les interactions qui ont lieu au cours de stages ou de colloques avec des praticiens étrangers suscitent et structurent aussi les aspirations à l'origine de la dynamique des métiers.

Si les premières revendications des restaurateurs français et américains sont analogues en termes de contenu et qu'elles perturbent effectivement le champ d'activité dans les deux pays, elles n'ont cependant pas la même issue. Dès les années 50, les conservateurs de musées

${ }^{8}$ Bulletin de liaison de l'Association des Elèves et des Anciens Elèves de l'IFROA, $\mathrm{n}^{\circ}$ 16, 1987, p. 18. américains commencent à reconnaître la spécificité de l'expertise des conservators, ce qui se traduit par le développement, au sein des musées, d'une division du travail horizontale, pluricéphale, dans laquelle les restaurateurs s'occupent de la conservation matérielle des œuvres et les conservateurs de leur mise en valeur. Les deux professions sont d'ailleurs aujourd'hui davantage occupées à lutter avec d'autres groupes naissants : les restaurateurs avec les scientifiques de laboratoire qui analysent les œuvres (conservation scientists) et les responsables des réserves et du mouvement des œuvres (collections managers); les conservateurs avec les administrateurs, les professionnels de l'éducation ou encore les designers intervenant lors de la préparation des expositions. Liée à l'accroissement de l'activité des musées à partir des années 60 , l'explosion des effectifs du personnel s'accompagne ainsi de l'apparition de nouvelles tâches et de nouveaux groupes qui tous ont des aspirations professionnelles, de sorte que le système des professions des musées n'en finit pas de se redéfinir.

En France, la mobilisation des restaurateurs d'œuvres d'art intervient quand les conservateurs se sont déjà saisis de l'activité de restauration. Les conservateurs du musée du Louvre, avec quelques collègues européens, défendent, dès les années 50, l'importance du rôle de l'historien d'art, qui serait doté d'une " conscience historique et critique [...] qui fait défaut au technicien » (Philippot, 1967). Cette offensive répond justement à la montée en puissance des restaurateurs dans les musées anglo-saxons. Dans les années 80 , à la suite de la mobilisation inédite des restaurateurs, les conservateurs renforcent encore leurs prérogatives en matière de restauration en introduisant des modules pratiques dans leur cursus de formation pour être davantage en mesure de prendre des décisions de restauration. Surtout, ils créent un nouveau segment de la profession : les conservateurs spécialisés en restauration, qui ne sont pas chargés d'une collection mais administrent les services de restauration centraux et régionaux. La non-intégration des restaurateurs français dans les musées à des postes de salariés a certes été favorisée par la conjoncture politique et économique, peu favorable à la création de nouveaux postes de fonctionnaires, mais elle est aussi le fruit du système des professions : d'une part, les conservateurs français refusent de voir se développer une organisation pluricéphale qui pourrait 
menacer leur dominance au sein des musées ; d'autre part, le groupe des régisseurs, moins qualifiés que les restaurateurs diplômés, est venu occuper la nouvelle niche de la « conservation préventive » qu'ils convoitaient ${ }^{9}$. Il s'agit notamment de faire régulièrement des « constats d'état » des œuvres, à l'occasion des prêts et des expositions. S'occupant d'abord principalement du transport des œuvres, les régisseurs ont étendu leurs prérogatives à la faveur du développement de la conservation préventive. Ainsi, la dynamique professionnelle des restaurateurs d'œuvres d'art français - en l'occurrence ici le fait qu'il n'y a pas de poste de salarié correspondant à leur statut - a été contrainte par celles des autres groupes du champ d'activité.

\section{Le lieu de travail comme canal de régulation des professions?}

Nous avons également étudié ce qui se passe dans les organisations où exercent les praticiens, en l'occurrence ici les musées et les ateliers de restauration. $\mathrm{Ce}$ niveau d'analyse permet en effet d'observer au plus près l'apparition et la disparition des appellations de métiers que Didier Demazière (2008) invite à considérer comme des indices des dynamiques professionnelles. Cependant, l'étude des lieux de travail est rarement exploitée en sociologie des professions. Surtout, suivant l'approche proposée par Freidson (1984 [1970]), les travaux existants cherchent davantage à apprécier le statut d'un groupe professionnel en étudiant la position des praticiens dans le système de travail d'une institution, qu'à expliquer la forme de la dynamique suivie par le groupe en question. De même, s'il invite les chercheurs à considérer ensemble les deux niveaux de luttes et de revendications que sont les lieux de travail ("sphère informelle ») et le niveau des groupes professionnels (« sphère formelle »), Abbott (1988) n'étudie pas empiriquement leurs relations.

Les lieux de travail sont d'abord le théâtre de luttes intra-organisationnelles qui font naitre des aspirations professionnelles. Aux États-Unis comme en France, comme nous l'avons souligné plus haut, les premières

\footnotetext{
9 Cette niche s'est développée, dans les années 90, avec l'élaboration de nouveaux standards de conservation, touchant non plus seulement aux œuvres mais aussi à leur environnement (vitrine, caisse, réserves, salles d'exposition).
}

revendications collectives des restaurateurs d'œuvres d'art trouvent ainsi leur origine dans la frustration que chacun a pu éprouver en situation de travail avec les conservateurs. Le cas des États-Unis montre aussi que c'est au sein de certains musées que des négociations ont été menées par les restaurateurs pour la revalorisation de leur position, revalorisation qui a ensuite gagné la profession tout entière. Le Metropolitan Museum of Art à New York est ainsi une des premières institutions ayant créé, dès les années 40 , une position de conservator. Elle vient remplacer celle de restorer, utilisée jusqu'alors pour désigner les ouvriers qui s'occupent de l'état matériel des œuvres. Mais le changement ne touche pas seulement l'appellation, il recouvre également une revalorisation de statut. Les conservators travaillent d'abord dans les départements curatoriaux ceux voués à l'organisation d'exposition de peintures, puis petit à petit également de dessins et gravures, de costumes, et bientôt de tous les départements du musée - sous la direction du curator et de ses adjoints (associate curators et assistant curators), puis comme équivalent du curator. Le conservator a alors lui aussi des positions subalternes : associate conservator, assistant conservator et conservation assistant. Enfin, à partir des années 70 , les conservators, tous grades confondus, deviennent indépendants lorsqu'est créé un département de conservation, composé d'un laboratoire pour les peintures, d'un autre pour les objets, et d'autres encore pour les dessins et les costumes. $\mathrm{Si}$ certains restorers conservent ce statut, inférieur à celui de conservation assistant, jusqu'à la retraite, les plus jeunes deviennent à leur tour conservators, en gravissant peu à peu tous les échelons de la nouvelle hiérarchie et en se formant sur le tas aux nouveaux standards. La revalorisation du groupe s'observe donc au sein même de l'organisation.

L'analyse des listes de personnel d'une dizaine de grands musées américains sur les cinquante dernières années montre que, systématiquement mais selon des temporalités différentes, les restaurateurs connaissent exactement cette même dynamique. Or, au Metropolitan Museum, la mise en place de la nouvelle hiérarchie, puis l'autonomisation de l'activité des restaurateurs au sein d'un département voué à la conservation sont le fruit de négociations entre les restaurateurs du musée et la direction. Et ce sont ces mêmes restaurateurs qui portent ensuite les 
revendications au niveau du groupe professionnel, travaillent à l'élaboration d'un code déontologique et à la création de formations spécialisées dans le cadre de l'AIC, et enfin cherchent à convaincre les directeurs de musées de se doter d'un laboratoire de conservation performant. Les lieux de travail constitueraient donc un canal de la régulation des groupes professionnels. Certains d'entre eux agissent comme des transformateurs du groupe professionnel, au sens où ils peuvent être à l'origine de l'apparition de postes de salariés et de nouveaux métiers, cristalliser de nouvelles relations hiérarchiques qui ensuite se diffusent à l'ensemble des organisations culturelles d'un pays. On comprend également que la relation entre les sphères formelle et informelle, évoquée plus haut, s'apparente à une boucle : les frustrations et les négociations qui ont cours au sein des organisations donnent lieu à des revendications qui s'expriment au niveau des groupes professionnels, et ces revendications peuvent ensuite venir redéfinir de manière générale les relations hiérarchiques sur les lieux de travail. C'est cette boucle qui n'a pas été effectuée dans le cas français, les restaurateurs d'œuvres d'art ayant manqué le tournant suscité par l'essor du secteur d'activité dans les années 70 et 80 .

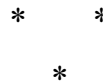

Expliquer la forme des dynamiques professionnelles nécessite donc de considérer un faisceau de facteurs touchant aussi bien aux contextes politique, économique et culturel dans lesquels elles s'inscrivent, qu'à la composition des associations professionnelles, aux relations d'interdépendance et de mimétisme liant les différents groupes impliqués dans l'activité, ou encore aux revendications et aux recompositions ayant lieu au sein même des lieux de travail. Les deux faisceaux de facteurs identifiés ici - les institutions d'un côté, et les luttes professionnelles de l'autre - n'interviennent pas de la même façon dans la construction des dynamiques professionnelles. L'État et ses multiples figures, ainsi que la composition des organisations professionnelles, définissent les contours possibles à l'intérieur desquels la dynamique du groupe se développe. Mais ce sont les luttes interprofessionnelles et intra-organisationnelles qui activent effectivement la dynamique.

Pour mieux comprendre cet emboîtement, prenons l'exemple de la création, dans les années 80 , de la première revue de restaurateurs d'œuvres d'art en France - un important jalon dans un processus de professionnalisation. En interrogeant le fondateur de la revue et ses collègues de l'époque, nous comprenons que ce qui a motivé cette initiative est la frustration éprouvée face à l'utilisation, par les conservateurs de musées, de leurs rapports de restauration, pour des publications et des conférences. Les restaurateurs concernés se côtoyaient régulièrement, notamment dans le cadre de la formation continue de l'Institut de restauration. Mais l'État a aussi joué un rôle important à l'époque, car c'est une délégation du ministère de la Culture qui a financé le développement de cette revue, dans le cadre d'une politique soutenant spécifiquement les métiers d'art. Deux facteurs au moins sont donc intervenus dans la reconnaissance de la dimension intellectuelle de l'activité des restaurateurs : d'une part, les interactions problématiques sur le lieu de travail et les échanges au sein des formations, qui activent la dynamique, et d'autre part les politiques publiques qui rendent possible et cadrent le développement de cette dynamique. Aux États-Unis, la création de la première revue sur la restauration est le fait de deux restaurateurs du Fogg Art Museum, qui bénéficient de fonds privés et du soutien des conservateurs du musée. Nous avons donc affaire à une tout autre construction, également activée par des interactions sur le lieu de travail, mais n'impliquant pas l'intervention des instances politiques - une construction marquée avant tout par l'empreinte des organisations muséales, là où celle observée en France se situait en dehors.

Il nous faudrait approfondir l'analyse des processus concrets à l'origine de la mobilité des métiers, et celle des rôles respectifs des institutions et des organisations. Cela nous permettrait de dépasser la comparaison terme à terme et d'en tirer toutes les conséquences quant à la compréhension des professions et de leur dynamique comme construction sociétale. 


\section{Bibliographie}

Abbott A. (1988), The System of Professions. An Essay on the Division of Expert Labor, Chicago, The University of Chicago Press.

Chapoulie J.-M. (1973), « Sur l'analyse sociologique des groupes professionnels ", Revue française de sociologie, vol. 14, pp. 86-114.

DiMaggio P.J., Powell W.W. (1983), “The Iron Cage Revisited: Institutional Isomorphism and Collective Rationality in Organizational Fields", American Sociological Review, vol. 48, n 2, pp. 147-160.

Demazière D. (2008), « L'ancien, l'établi, l'émergent et le nouveau : quelle dynamique des activités professionnelles? », Formation Emploi, n ${ }^{\circ}$ 101, pp. 41-54.

Dubar C. et Tripier P. (1999), Sociologie des professions, Paris, Armand Colin.

Freidson E. (1984 [1970]), La profession médicale, Paris, Payot.

Freidson E. (1986), « Les professions artistiques comme défi à l'analyse sociologique ", Revue française de sociologie, vol. 28, pp. 431-443.

Freidson E. (1994), « Pourquoi l'art ne peut pas être une profession ", in Menger P.-M. et Passeron J.-C., L'art de la recherche : essais en l'honneur de Raymonde Moulin, Paris, La Documentation française.

Freidson E. (2001), Professionalism: the Third Logic, Chicago, The University of Chicago Press.

Halliday T.C. (1987), Beyond Monopoly : Lawyers, State Crises, and Professional Empowerment, Chicago, University of Chicago Press.

Heidenheimer A.J. (1989), "Professional knowledge and state policy in comparative historical perspective: law and medicine in Britain, Germany and the United States", International Social Science Journal, $\mathrm{n}^{\circ} 122$, pp. 529-53.
Hénaut L. (2008), « Montée en qualification et perte de contrôle. Les restaurateurs de tableaux et leurs documents de travail ", in Observer le travail. Histoire, ethnographie, approches combinées, Arborio A.-M., Cohen Y., Fournier P., Hatzfeld N., Lomba C. et Muller S. (dir.), Paris, La Découverte, coll. « Recherches », pp. 95-112.

Le Bianic T. (2008), « L'État et les professions dans la sociologie continentale et anglo-américaine : un état des lieux ", in Le Bianic T. et Vion A., Action publique et légitimité professionnelle, LGDJ, Paris, pp. 249-277.

Le Feuvre N. et Walters P. (1994), "The Legal Professions in France and Britain: Comparative Perspective on the Dynamics of Occupational Feminisation", in Dubar C. et Lucas Y. (dir.), Genèse et dynamique des groupes professionnels, Lille, Presses Universitaires de Lille, Coll. « Mutations/ Sociologie », pp. 343-356.

Martel F. (2006), De la culture en Amérique, Paris, Gallimard.

Marry C. (2004), Les femmes ingénieurs, une révolution respectueuse, Paris, Belin, Coll. " Perspectives sociologiques ».

Ministère de la Culture et de la Francophonie (1994), « La profession de restaurateur d'œuvres d'art », Développement culturel, Bulletin du Département des Études et de la Prospective, ${ }^{\circ}$ 104, juin, pp. 1-6.

Percival-Prescott W. (2003), "Foreword: Origins and Outcome of the Lining Conference", in Villers C. (dir.), Lining paintings: papers from the Greenwich Conference on Comparative Lining Techniques, London.

Philippot P. (1967), « Le problème des relations entre historiens d'art et restaurateurs ", Communication présentée au colloque de Venise, Les responsabilités de l'historien dans la conservation et la restauration 
des monuments et œeuvres d'art; texte publié dans le Bulletin du congrès International d'Histoire de l'Art, vol. 2, pp. 8-9.

Rebérioux M. (1990), « Les métiers d'art, ou le pouvoir de l'État Républicain ", Technologies, Idéologies et Pratiques, vol. 9, n 3-4, pp. 119-134.

Stout G. (1950), "The Viewpoint of the Conservator",
Alumni, vol. 19 ; cité en français par H. C. von Imhoff dans son introduction au « Texte de définition de la profession » in Les nouvelles de l'ICOM, vol. 39, $\mathrm{n}^{\circ} 1,1986$, pp. $2-4$.

Zarca B. (1988), « Identité de métier et identité artisanale », Revue française de sociologie, vol. 29, pp. 247-273.

\section{Résumé}

\section{La construction des groupes professionnels : le cas des restaurateurs d'œuvres d'art en France et aux États-Unis}

Léonie Hénaut

En comparant les chemins de professionnalisation empruntés à partir des années 60 par les restaurateurs d'œuvres d'art en France et aux États-Unis, cet article étudie les acteurs et les processus intervenant dans la construction des groupes professionnels. Les institutions, comme l'État et les associations professionnelles, contribuent certes à déterminer les contours possibles des dynamiques professionnelles - établissement d'un monopole sur le marché des musées pour les diplômés des nouvelles formations spécialisées en France, absence de règlementation et développement du salariat côté américain. Cependant, les luttes interprofessionnelles et les négociations intra-organisationnelles alimentent aussi ces dynamiques, en particulier celles qui touchent à la place des restaurateurs dans la division du travail par rapport aux conservateurs de musées.

Mots clés :

Restaurateur d'art, professionnalisation, organisation professionnelle, identité professionnelle, France, États-Unis

Journal of Economic Literature: J 44 